\title{
Development of an Adaptive IIR Filter Based on Modified Robust Mixed-Norm Algorithm for Adaptive Noise Cancellation
}

\author{
Ismaila Adeniyi Kamil* \\ Department of Electrical \& Electronic Engineering, University of Ibadan, Ibadan 200284, Nigeria \\ Taiwo Oyedotun Ajao \\ Department of Electrical \& Electronic Engineering, University of Ibadan, Ibadan 200284, Nigeria
}

\begin{abstract}
Noise cancellation is one of the most important applications of adaptive filters. The employment of adaptive filtering in most digital signal processing tasks is currently an area of growing interest as adaptive filters, due to their dynamic nature, perform better than the traditional filters in compensating for random noise in their environment. However, the compensation for impulsive interference or noise is desired since most adaptive algorithms earlier proposed modelled noise as a random process of the White Gaussian distribution. A modified robust mixed-norm (MRMN) algorithm recently proposed to compensate for impulsive interference has been found to be hardware efficient, however the MRMN algorithm has only been tested on adaptive FIR system identification task. In this paper, an adaptive IIR filter based on MRMN adaptive algorithm is proposed and tested for noise cancellation task. The developed filter structure was modelled and simulated in MATLAB environment. The results obtained showed that the MRMN algorithm does in fact compensate for the presence of impulsive interference, however, at a higher computational complexity relative to the LMS algorithm.
\end{abstract}

Keywords: Noise cancellation, adaptive filtering, impulsive noise, adaptive algorithm, system identification, random noise

DOI: $10.7176 / \mathrm{CEIS} / 10-2-01$

Publication date:March $31^{\text {st }} 2019$

\section{Introduction}

Noise-free output is usually a desired characteristic in many applications. The concept of noise cancellation has therefore been identified as a vital method to eliminate noise contained in useful signals in applications such as communications, image processing, biomedical engineering and speech enhancement (Vaseghi, 2000). Filtering is an essential tool to get desired signal in the presence of noise. If the prior knowledge of the signal is available, then the filter can be designed so that it passes the desired frequency and attenuate most of the unwanted frequency components (Parmar, et al., 2017). However, there are many digital signal processing applications in which the prior knowledge of signals cannot be specified such as channel equalisation, channel estimation, echo cancellation and system identification, hence, the concept and problem of adaptive filtering become relevant.

Adaptive filtering can be described as a method of filtering, in which dynamic (adaptive) filters with selfadjusting coefficients minimize a cost function by virtue of a pre-defined sets of (Apolinario Jr. \& Netto, 2009). The cost function is the difference in measure between the reference or desired signal and the output of the adaptive filter. Finite impulse response (FIR) and infinite impulse response (IIR) filters are the two types of digital filters that form the bedrocks of adaptive filters.

The FIR filters are routinely used in adaptive filtering applications and the reasons for their popularity include their inherent stability, faster convergence and availability of simple and efficient algorithms for adjusting the filter coefficients (Avalos, et al., 2011). However, IIR adaptive filters, if implemented in a stable realization, are more suitable for use in most adaptive filtering task e.g. in a system identification procedure, where the unknown system has high levels of recursion. It would take a higher order FIR adaptive filter, with numerous coefficients to approximate this particular system, whereas, a single or double pole IIR adaptive filter might be enough to complete the same task, hence, IIR adaptive filters are comparably cheaper and more reasonable to implement in such cases (Netto, et al., 1995). However, some of the drawbacks of IIR adaptive filters include algorithm instability, slow convergence and convergence to biased and/or local minimum solutions (Apolinario Jr. \& Netto, 2009). Consequently, several new algorithms for IIR adaptive filtering have recently been proposed to overcome these problems.

The least mean square (LMS) has been the traditional algorithm for adaptive filtering and has gained acceptability for hardware implementation by several researchers due to simple structure (Dixit, 2017), (Gupta \& Beniwal, 2015). The main weakness of the conventional type LMS lies in its complexity in selectin. The main weakness of the conventional type LMS lies in its complexity in selecting a suitable value for the step size parameter that guarantees stability. It has also been shown that the algorithm converges fast for Gaussian noise but fails to converge for impulsive noise. To overcome these and other problems inherent in the conventional LMS, several variants of the algorithm have been proposed among which are time-varying LMS (TV-LMS), normalized 
LMS (NLMS), modified normalized LMS (MN-LMS), leaky LMS (LLMS), block LMS (BLMS), sign-error LMS (SE-LMS), sign-data LMS (SD-LMS), sign-data normalized LMS (SDN-LMS), sign-sign LMS (SS-LMS), signsign LMS algorithm with leakage term (SS-LMS-LT), variable step-size LMS (VS-LMS), filtered-x-LMS (FxLMS), frequency response shaped LMS (FRS-LMS) and hybrid LMS (H-LMS).

More recently a robust mixed-norm (RMN) adaptive filter algorithm was proposed that is suitable for system identification applications in which the desired response is corrupted by additive impulsive (Chambers \& Avlonitis, 1997). The algorithm which is combination of the conventional LMS and SE-LMS has been shown to be robust to impulsive noise.

The use of constant step size in the RMN algorithm leads to slow and generally non-uniform convergence. To overcome this problem, a normalized robust mixed-norm (NRMN) algorithm was proposed which replaces the constant step size, $\mu$ in the RMN with a variable one, $\mu(\mathrm{k}$ (Papoulis \& Stathaki, 2004).

Although the robust mixed-norm algorithms are immune to impulsive noise, they involve high computational cost there to their level of complexity. To overcome the complexity issue in the RMN algorithm a modified robust mixed-norm (MRMN) algorithm was proposed (Parmar, et al., 2017). The MRMN solved the complexity problem by employing a constant switching factor, $\alpha$ which is proportional to the standard deviation, $\sigma$ of the error signal to switch between the LMS and SE-LMS algorithms.

In this paper, the focus is on developing an IIR adaptive filter which is capable of additive impulsive noise cancellation. The developed filter is based on the MRMN which robust to additive impulsive noise with low complexity.

\section{Review of Related Works}

The basic configuration of an adaptive filter in discrete-time domain is illustrate in Figure 1 in which the reference input signal is denoted by $x(k)$, the desired output signal by $d(k)$ and the adaptive filter output signal by $y(k)$ (Apolinario Jr. \& Netto, 2009). The error signal which the difference between the desired signal and filter output is given by

$$
e(k)=d(k)-y(k)
$$

The adaptation algorithm uses $\mathrm{e}(\mathrm{k})$ to update the adaptive filter coefficients $\boldsymbol{w}(k)$ in accordance with some performance criteria. The LMS algorithm is the earliest adaptation algorithm employed for adaptive filtering task. The LMS algorithm is based on the gradient search with the weight vector update equation given as

$$
\boldsymbol{w}(k+1)=\boldsymbol{w}(k)+\mu e(k) \boldsymbol{x}^{*}(k)
$$

The high acceptance of this classical optimization approach in adaptive filtering is due to its low complexity and simplicity of implementation (Upadhyay, et al., 2016). However, this technique suffers from slow convergence speed to reach the optimal solution.

Several variants of the conventional LMS algorithms have been developed to improve on the speed of convergence. One of such algorithms is the Least Mean Mixed Norm (LMMN) which combines the advantages of different error norms (Chambers, et al., 1994); Tanrikulu \& Chambers, 1996) such as the conventional, relatively well-behaved LMS algorithm and the more sensitive, but better converging least mean fourth (LMF) algorithm. The algorithm is based on the minimisation of

$$
J(k)=\lambda E\left\{e^{2}(k)\right\}+(1-\lambda) E\left\{e^{4}(k)\right\}
$$

where $\mathrm{k}$ is the sample index and $\mathrm{E}($.$) is the mathematical expectation operator and \lambda \epsilon[0,1]$ is the mixing parameter. The LMMN weight update equation is given as

$$
\boldsymbol{w}(k+1)=\boldsymbol{w}(k)+2 \mu e(k)(\lambda+2(1-\lambda)) e^{2}(k) \boldsymbol{x}(k)
$$

The convergence properties of this algorithm are controlled by $\lambda$ and $\mu$, the adaptation gain parameter.

A robust mixed-norm algorithm (RMN) reported in Chambers \& Avlonitis (1997) combines the conventional LMS and SE-LMS stochastic gradient algorithms. The motivation for this algorithm rests on the fact that for identical initial convergence rates, the LMS provides a more accurate final solution, less misadjustment in the absence of impulsive noise, but is very sensitive to the presence of outliers; whereas, with fixed $\mu$, the SE-LMS algorithm is less accurate, with higher misadjustment, but is more robust to the presence of outliers. The RMN is based on the minimization of the following error norms

while the weight update equation is expressed as

$$
J(k)=\lambda E\left\{e^{2}(k)\right\}+(1-\lambda) E\{|e(k)|\}
$$

$$
\boldsymbol{w}(k+1)=\boldsymbol{w}(k)+\mu\{\lambda 2 e(k)+(1-\lambda) \operatorname{sign}[e(k)] \boldsymbol{x}(k)\}
$$

Another variant of the RMN, the normalized RMN (NRMN) was proposed in Papoulis \& Stathaki (2004) in which the constant step size $\mu$ had been replaced with a variable one $\mu(\mathrm{k})$, which takes into account the instantaneous tap-input-vector power $\|\boldsymbol{x}(k)\|_{2}^{2}$ and the current value of $\lambda(\mathrm{k})$ and $\mathrm{e}(\mathrm{k})$.

Although the robust mixed-norm algorithms are designed to suppress the impulsive noise, the calculation of $\mu$ and $\lambda$ results in high computational cost and this makes it unsuitable for hardware implementation. The modified RMN (MRMN) reported in (Parmar, et al., 2017) uses a switching constant, $\alpha$ to switch between the weight update 
equations of the LMS and SE-LMS algorithms under the conditions stated in equation (7).

$$
\boldsymbol{w}(k+1)= \begin{cases}\boldsymbol{w}(k)+\mu \operatorname{sign}[e(k)] \boldsymbol{x}(k), & |e(k)|>\alpha \\ \boldsymbol{w}(k)+\mu e(k) \boldsymbol{x}(k), & |e(k)| \leq \alpha\end{cases}
$$

where $0<\mu<2 / \lambda_{\max }$, and $\alpha=3 \sigma ; \lambda_{\max }$ is the maximum value of the auto correlation matrix of input $\boldsymbol{x}(k)$ and $\sigma$ is the standard deviation of the error signal.

One of the adaptive IIR filtering LMS algorithms that has been found to be relatively stable and simple to implement is the Feintuch's LMS algorithm (Rupp, 2015; Stewart, 2006). The adaptive filter based on this algorithm is easily implemented using an IIR filter realization based on two transversal FIR filters as illustrated in Figure 1.

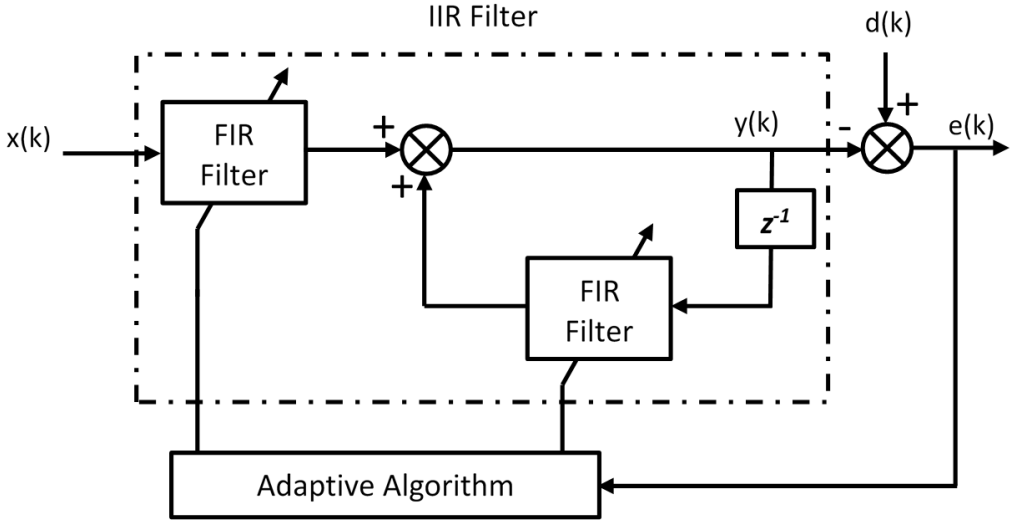

Figure 1. A two-transversal filter model of an IIR adaptive filter

The IIR filter structures with $N$ and $M-1$ feedforward and feedback weights, respectively, in the time domain, is described by the input-output relationship

The equation (8) in vector form is

$$
y(k)=\sum_{n=0}^{N-1} a_{n} x(k-n)+\sum_{m=1}^{M-1} b_{m} x(k-m)
$$

$$
\begin{gathered}
y(k)=\boldsymbol{a}^{T} \boldsymbol{x}(k)+\boldsymbol{b}^{T} \boldsymbol{y}(k-1)=\boldsymbol{w}^{T} \boldsymbol{u}(k) \\
\boldsymbol{w}^{T}=\left[\boldsymbol{a}^{T}, \boldsymbol{b}^{T}\right]=\left[\begin{array}{c}
\left.a_{0}, a_{1}, \ldots \ldots, a_{N-1}, b_{1}, b_{2}, \ldots \ldots, b_{M-1}\right] \\
\boldsymbol{u}^{T}=\left[\boldsymbol{x}^{T}(k), \boldsymbol{y}^{T}(k-1)\right]
\end{array}\right. \\
=[x(k), x(k-1) . . x(k-N+1), y(k-1), y(k-2) . . y(k-M+1)]
\end{gathered}
$$

The weight update equation using Feintuch's LMS algorithm is

$$
\boldsymbol{w}(k+1)=\boldsymbol{w}(k)+\mu e(k) \boldsymbol{u}^{*}(k)
$$

Noise cancellation is one of the most important applications of adaptive filters, in which it desired to recover a useful signal, $s(k)$ from a noisy one, $s(k)+n(k)$ (Dixit, 2017; Lee, et al., 2017; Qureshi, et al., 2017). In adaptive noise cancellation, the noisy signal, $s(k)+n(k)$ is employed as the reference signal for the adaptive filter whose input must be $\hat{n}(k)$, another version of the noise signal which is strongly correlated to $n(k)$ as illustrated in Figure 2. The adaptive mechanism adjusts the filter coefficients in such a manner that the filter output $y(k)$ approximates $n(k)$, thus forcing the error signal, $e(k)$ to resemble signal $s(k)$ (Apolinario Jr. \& Netto, 2009). 


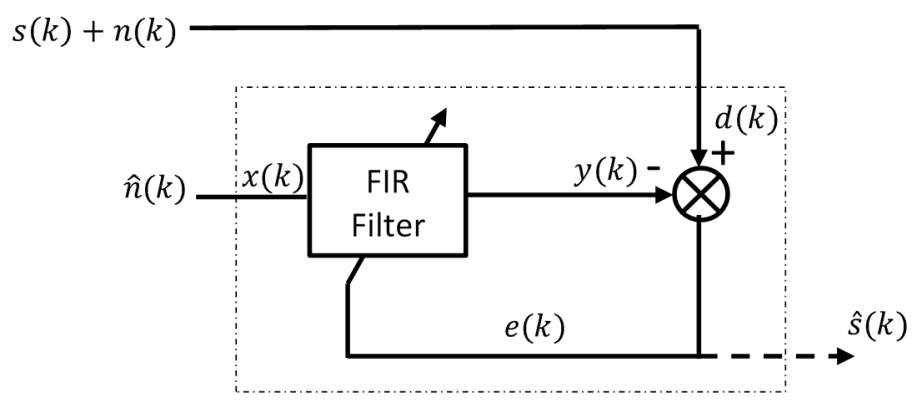

Figure 2. Adaptive Noise Cancellation

Most of the recent works in adaptive noise cancellation employed FIR adaptive filter structure based on either traditional LMS or one of its numerous (Chhikara \& Singh, 2012; Lee, et al., 2017; Thakkar, 2017; Huang, et al., 2017). These approaches have been reported to be inefficient in the presence of impulsive noise.

The proposed work employs an IIR adaptive filter structure based on the MRMN adaptive algorithm which a combination of Feintuch's LMS algorithm and Sign-Error Feintuch's LMS algorithm. The use Feintuch's LMS algorithm is to overcome as much as possible the stability problem associated with IIR filter while the MRMN will make the filter efficient in the presence of impulsive noise.

\section{Materials and Method}

\subsection{Proposed method}

This work employs the use of the MRMN algorithm in an IIR filter configuration, by using the Feintuch's LMS as a replacement for the traditional LMS which is more optimized for FIR adaptive filter implementation through the use of equations (7) to (10). The Sign-Error LMS is also modified in the same manner i.e. the weight update vector, for both the feedforward and recursive filters use the sign of the error signal (instead of the actual value) in its computation. The developed system for noise cancellation is illustrated in Figure 3.

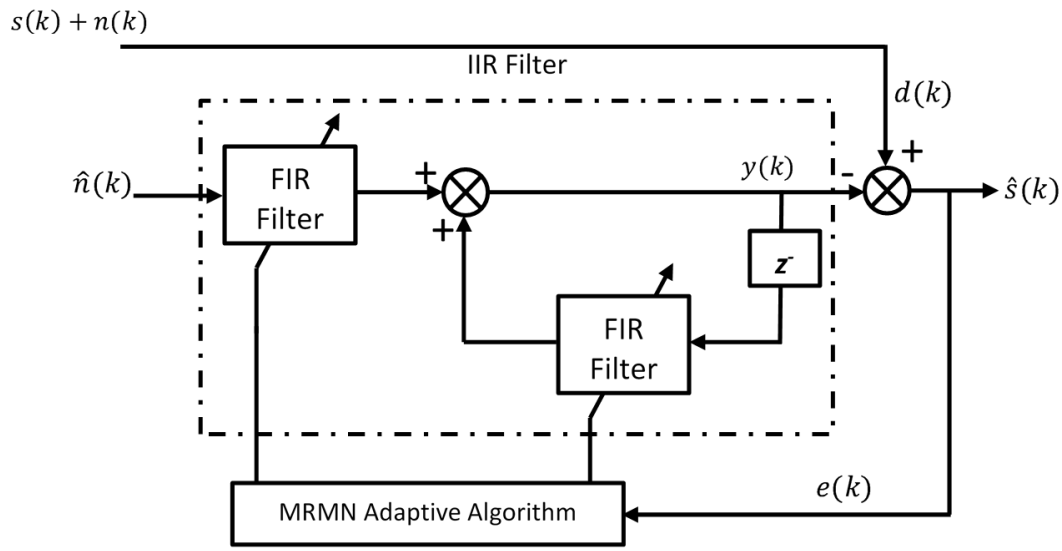

Figure 3. Proposed IIR Adaptive Noise Canceller

\subsection{Procedure of Design and Simulation}

The system in Fig.3 was modelled in MATLAB environment. Two sets of simulations were carried out - using Additive White Gaussian Noise as the noise signal, and using Additive Impulsive Noise. The following procedures were followed in the simulation process

(i). Generate a random sine wave signal, $s(k)$ as the original signal to be recovered.

(ii). Create a noise signal $n(k)$, which is AWGN to corrupt the original signal. The AWGN should have zero mean and standard deviation of 1 .

(iii). Initialize the desired/reference signal, $d(k)$ by adding the noise signal generated to the original signal i.e. $d(k)=s(k)+n(k)$.

(iv). The initial noise signal is passed through a first order lowpass filter to generate a reference noise, $\hat{n}(k)$, which is highly correlated with the input noise, $n(k)$, and uncorrelated with the sine wave signal, $s(k)$. This serves as the input to the adaptive filter.

(v). Initialize the error signal variable, $e(k)$ and adaptive filter output variable $y(k)$, (which should be of the same length as the original signal).

(vi). Initialize the adaptive filter weight vectors, $\boldsymbol{w}(\mathbf{0})$ according to equation $(9-10)$, with initial values of zero. 
(vii). Calculate the value of $\alpha$ is as described in equation (7). After the error analysis, a constant value for $\alpha$ is then chosen.

(viii). Starting with the initial values of all the variables, run the simulation for 500 iterations (i.e. $k=500)$

(ix). The error signal generated by the adaptive filter serves as the best approximation of the original signal, and it is plotted.

(x). The location of the poles and zeroes of the filters are calculated from the filters final transfer function to determine their stability.

(xi). The procedures were repeated with FIR adaptive filter using traditional LMS algorithm, FIR adaptive filter using MRMN algorithm and IIR adaptive filter using traditional LMS algorithm. Performance indicators, such as signal recovered, convergence speed, stability and system complexity are used for comparison.

The procedures are repeated using Additive Impulsive Noise which is a mix of AWGN and impulsive noise as the noise signal to corrupt the original signal. The impulsive noise is a multiplicative model of a binary process i.e. 1 and 0 with a total occurrence probability of 1 , with a stationary process/signal with fixed amplitude.

\section{Results and Discussion}

The simulation results for different adaptive noise cancellation when the signal is corrupted by AWGN are illustrated in Figures 4 to 13 while the percentage signal recovery and recovery speed are illustrated in Table 1.

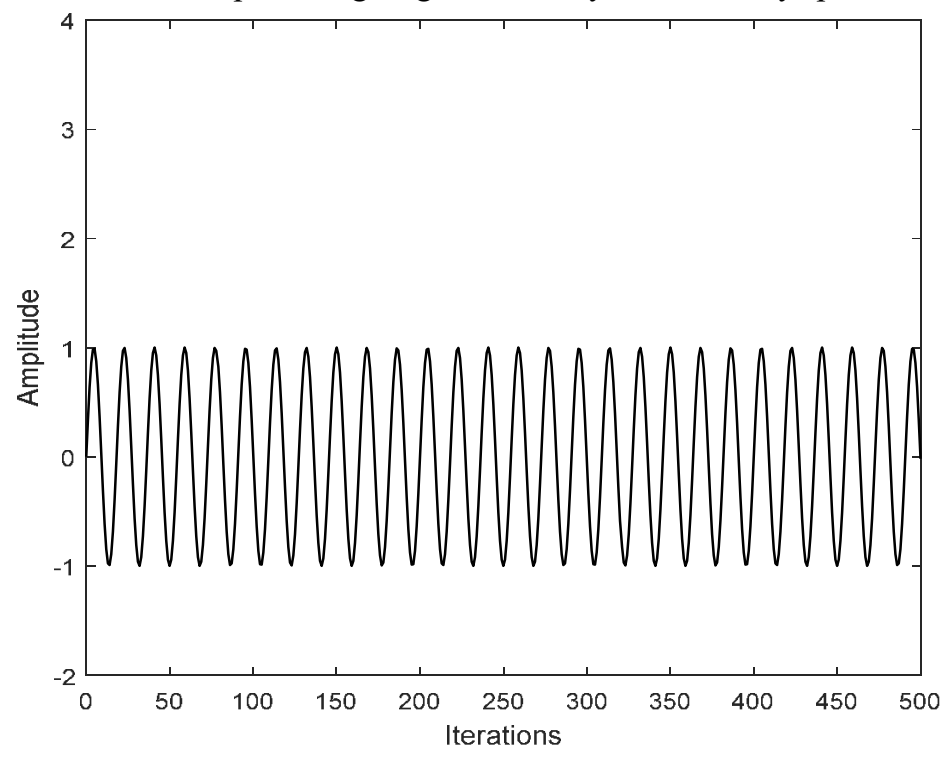

Figure 4. Original Uncorrupted Signal

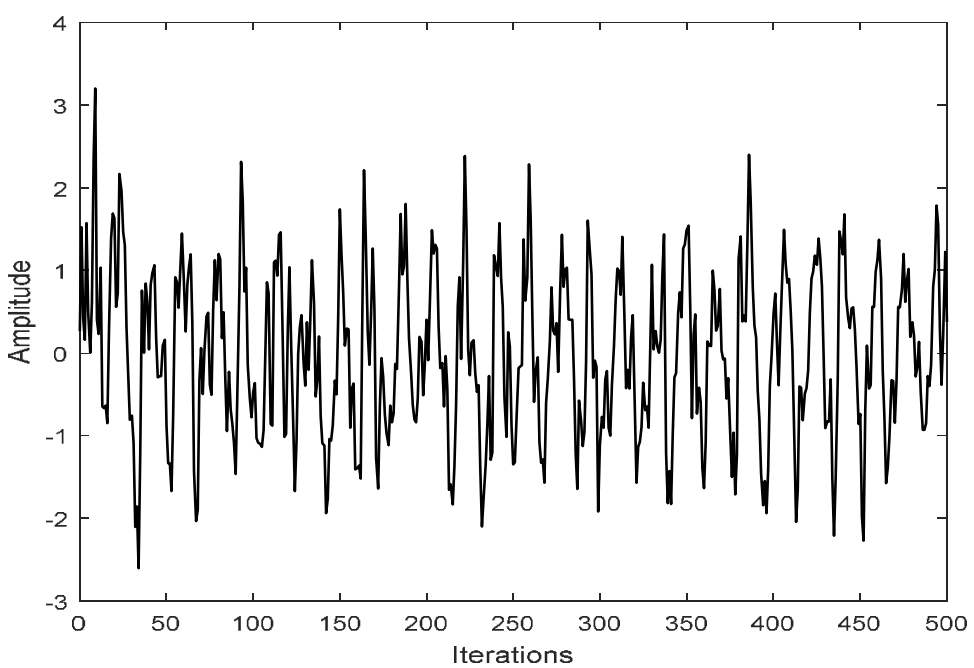

Figure 5. Signal Corrupted with AWGN 


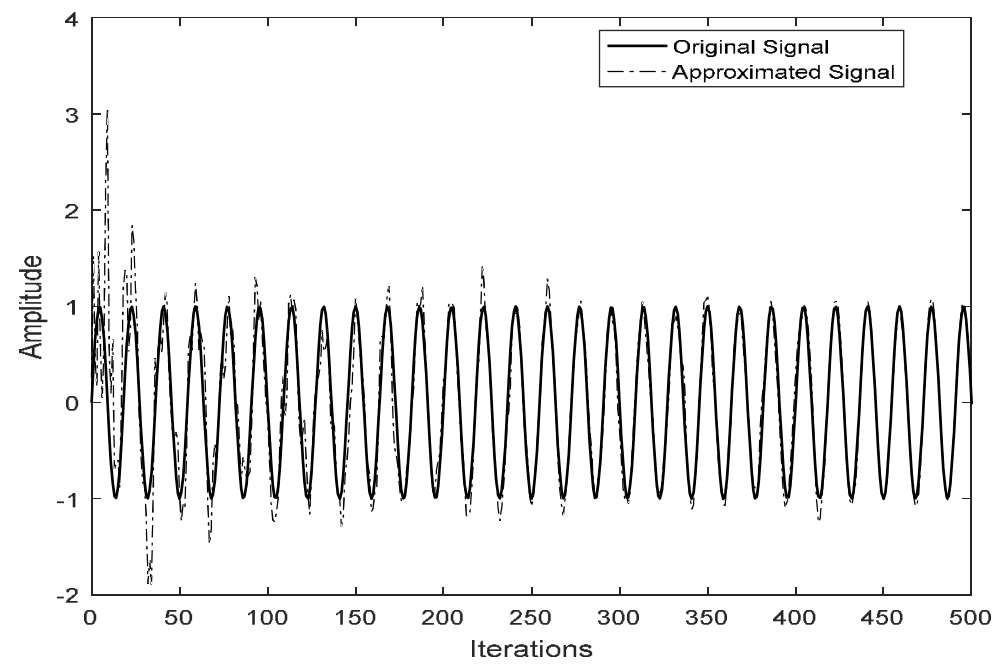

Figure 6. Signal Recovery from AWGN with FIR-LMS Filter

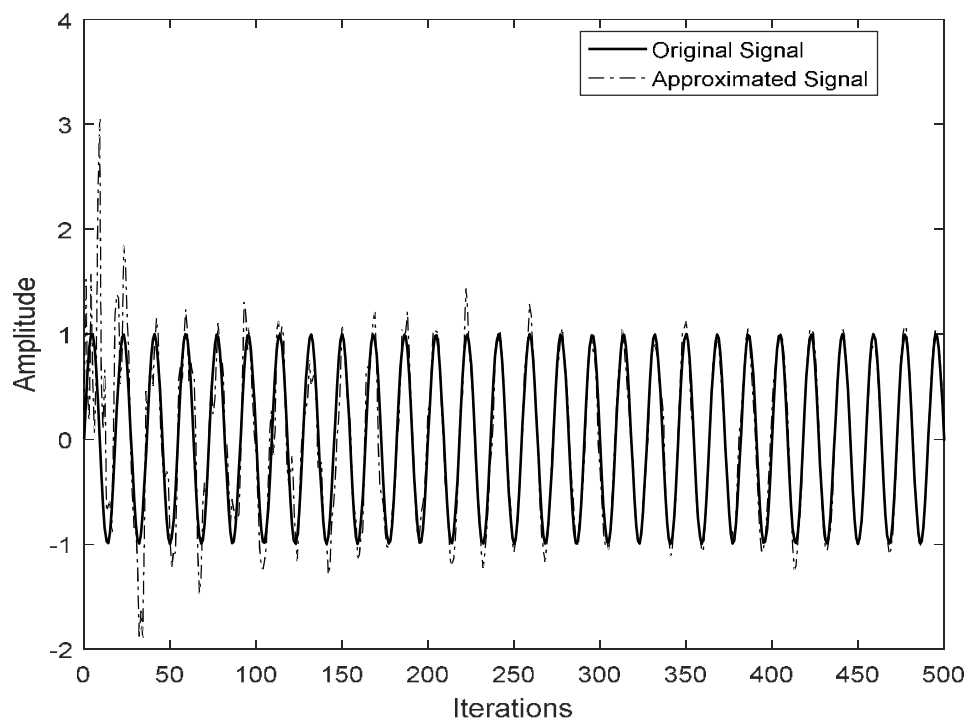

Figure 7. Signal Recovery from AWGN with FIR-MRMN Filter

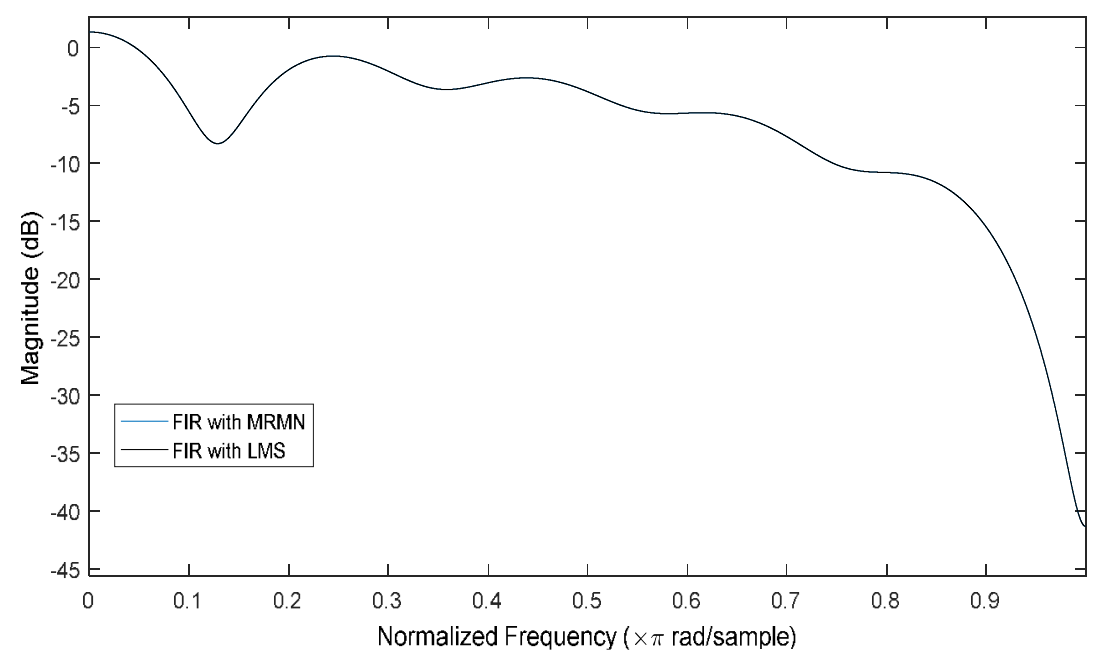

Figure 8. FIR Filter Response for Signal Recovery from AWGN 


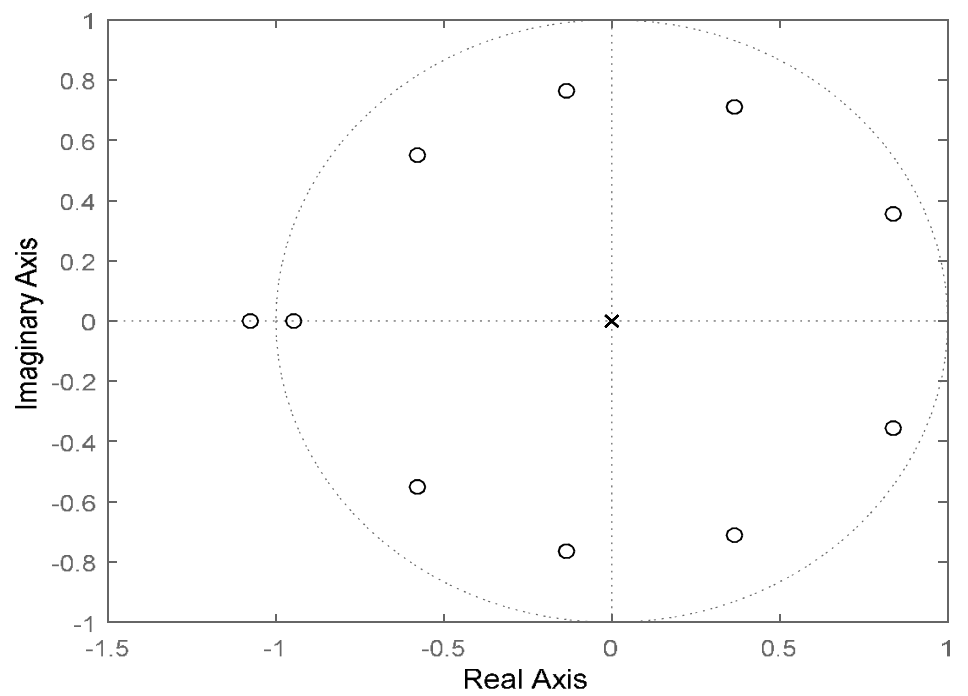

Figure 9. FIR Filter Pole-Zero Plot for Signal Recovery from AWGN

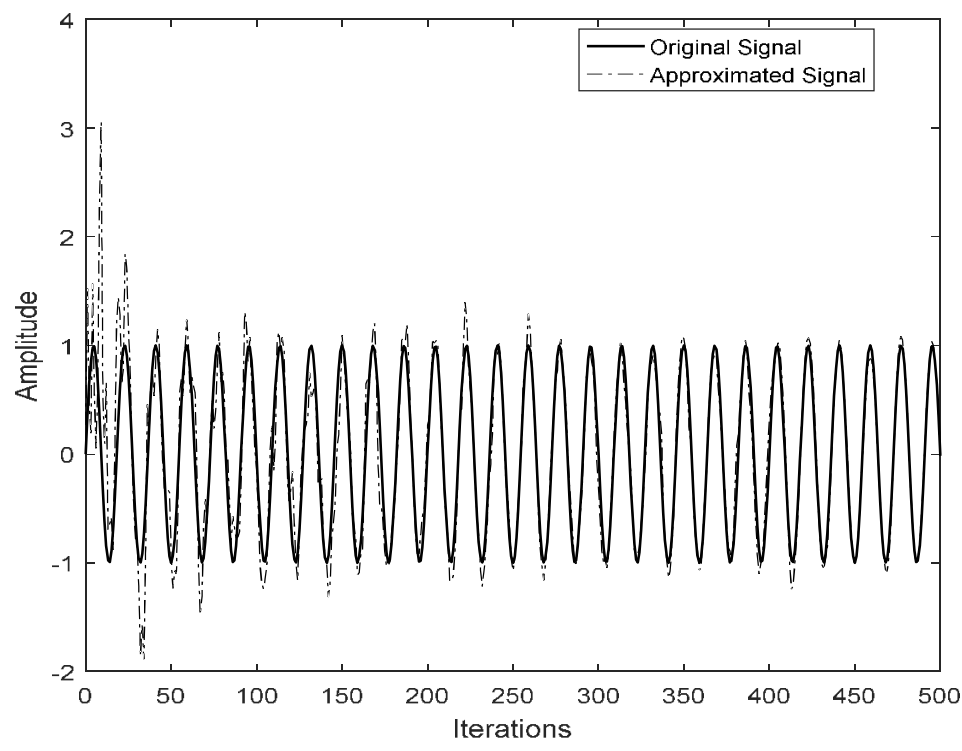

Figure 10. Signal Recovery from AWGN with IIR-LMS Filter 


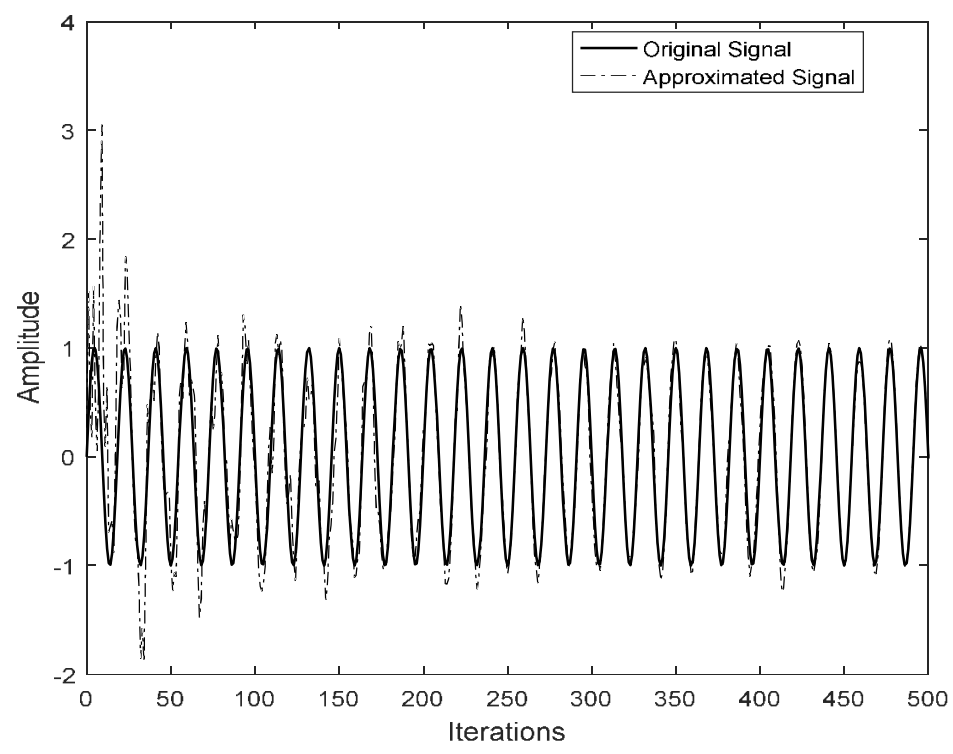

Figure 11. Signal Recovery from AWGN with IIR-MRMN Filter

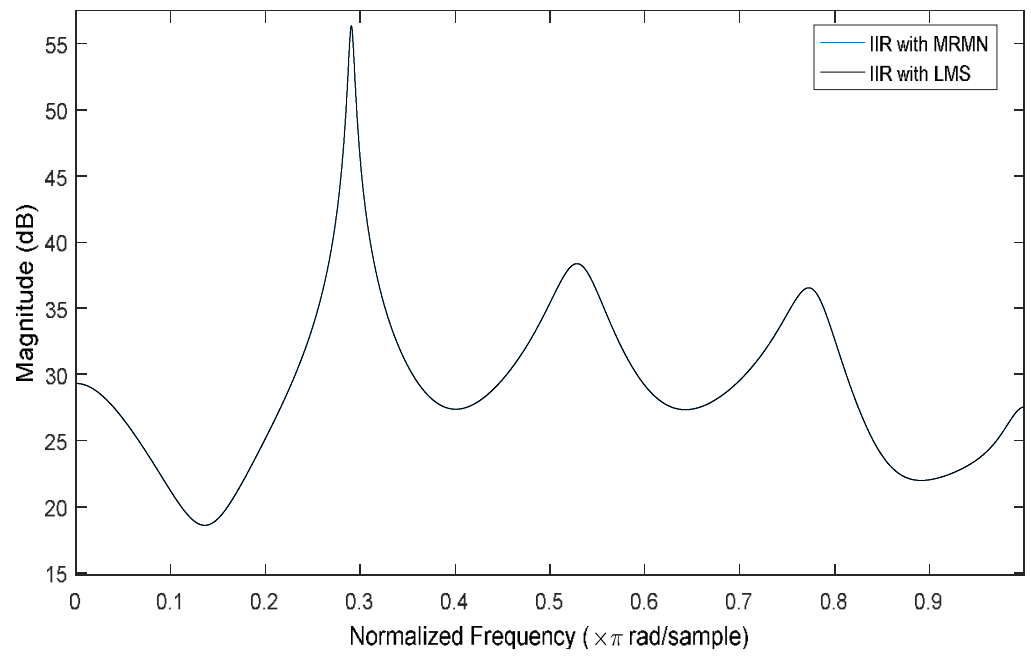

Figure 12. IIR Filter Response for Signal Recovery from AWGN 


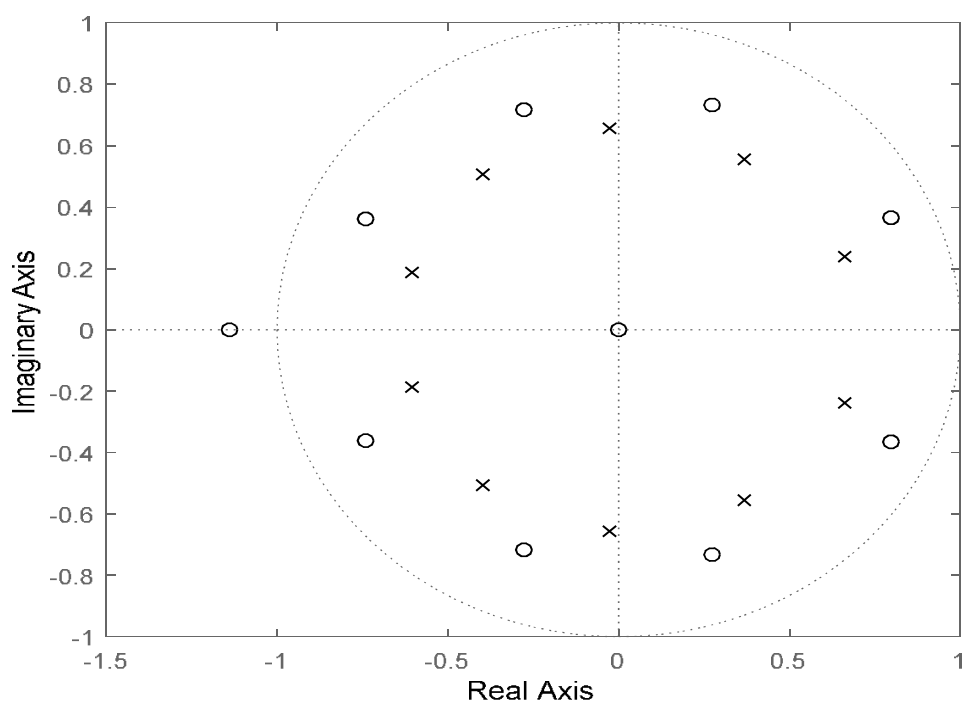

Figure 13. IIR Filter Pole-Zero Plot for Signal Recovery from AWGN

Table 1: Percentage Signal Recovery and Recovery Speed from AWGN

\begin{tabular}{ccc}
\hline \hline Filter Type & $\begin{array}{c}\text { Percentage Signal } \\
\text { Recovery }\end{array}$ & Speed of Recovery \\
\hline FIR-LMS & 94.6470 & 0.001088 \\
FIR-MRMN & 94.6470 & 0.011753 \\
IIR-LMS & 94.6776 & 0.003516 \\
IIR-MRMN & 94.6776 & 0.021727 \\
\hline
\end{tabular}

As observed from Figures $4-13$ and Table 1, all the filters had approximately similar performances in terms of convergence and signal approximation, which is due to the fact that, under strictly white gaussian noise conditions, the MRMN algorithm ideally converges to LMS algorithm, as the need to remove outliers using the SE-LMS algorithm is absent. However, filters with LMS algorithm were relatively faster in computation than the MRMN filters. This is due to low complexity of the LMS algorithm as compared with the MRMN.

The simulation results obtained when the noise is additive impulsive noise are illustrated in Figures $14-22$ and Table 2.

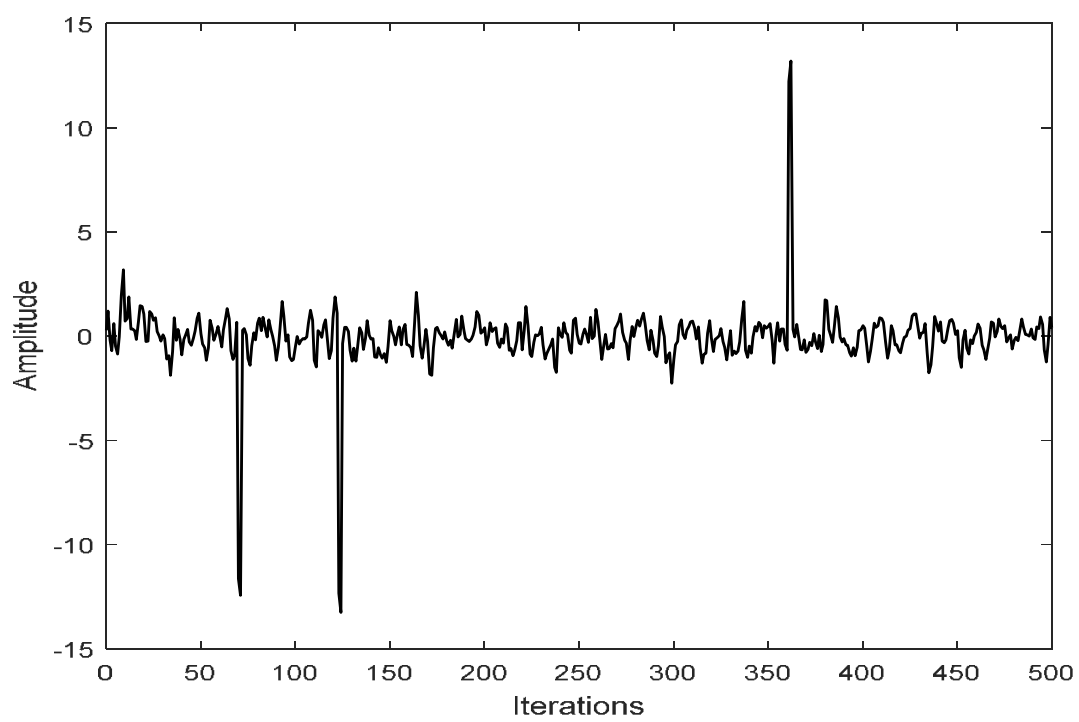

Figure 14. Signal Corrupted with Impulsive Noise 


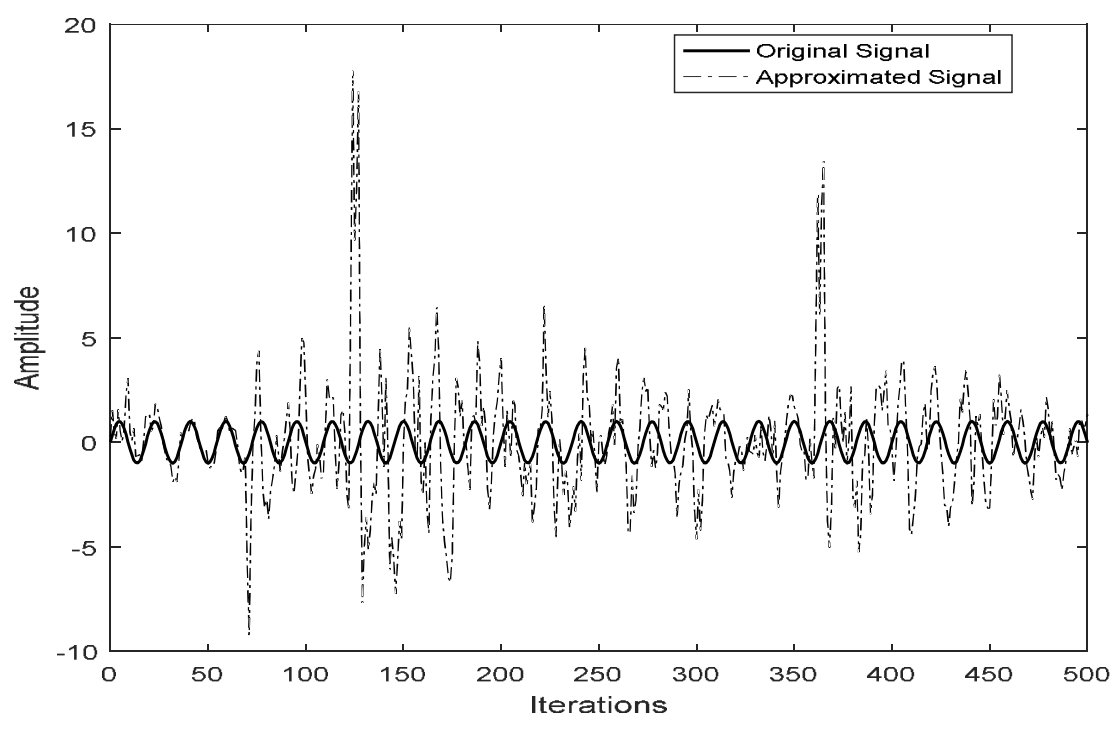

Figure 15. Signal Recovery from Impulsive Noise with FIR-LMS Filter

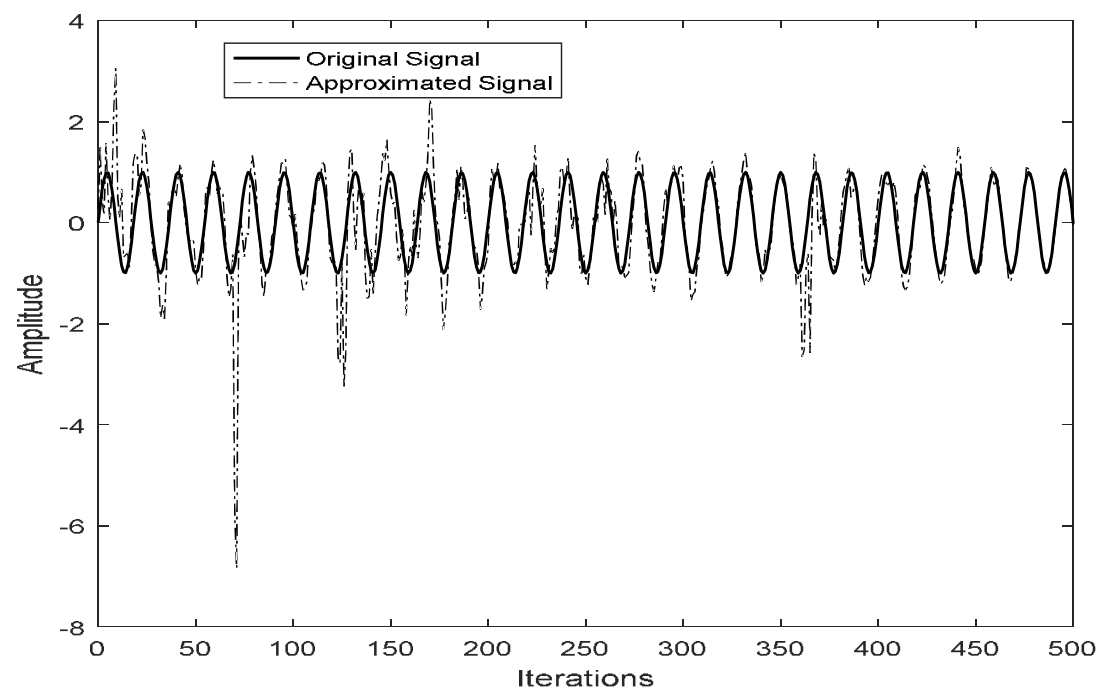

Figure 16. Signal Recovery from Impulsive Noise with FIR-MRMN Filter

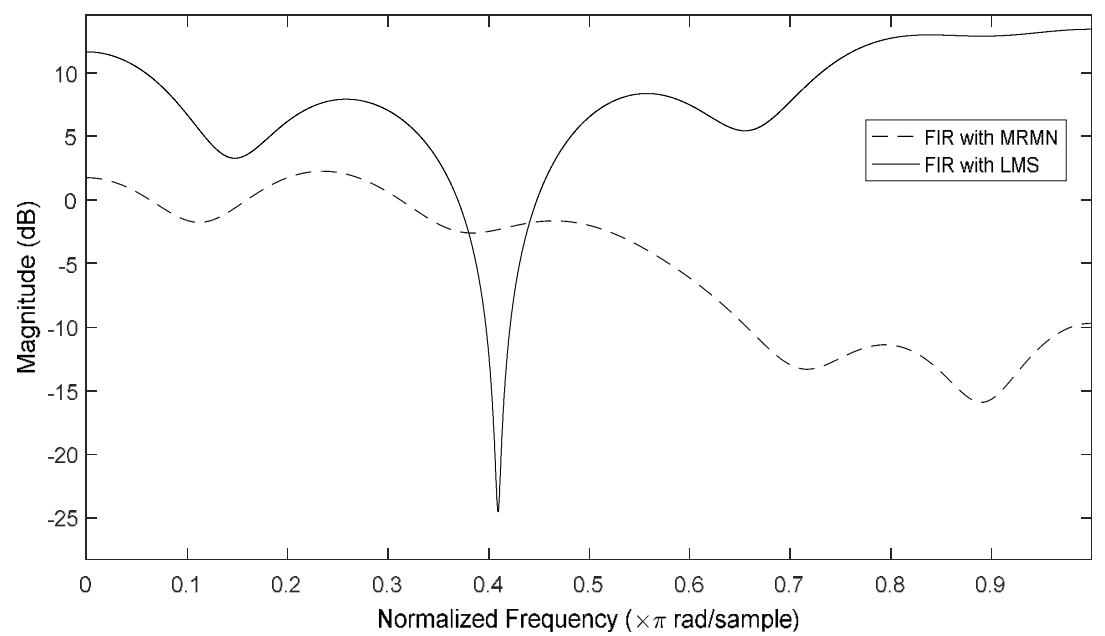

Figure 17. FIR Filter Response for Signal Recovery from Impulsive Noise 


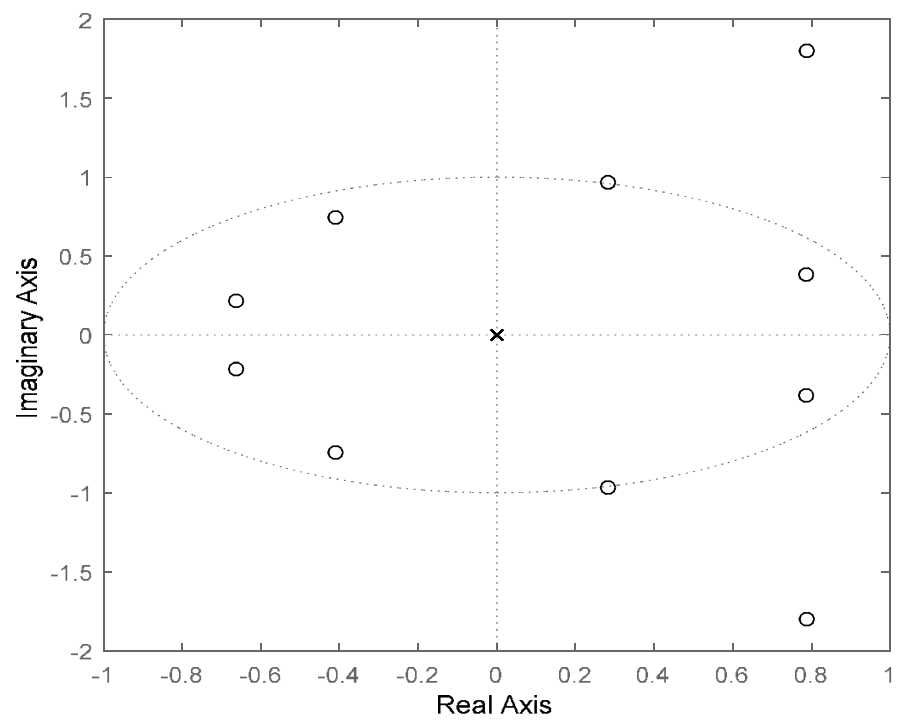

Figure 18. FIR-LMS Filter Pole-Zero Plot for Signal Recovery from Impulsive Noise

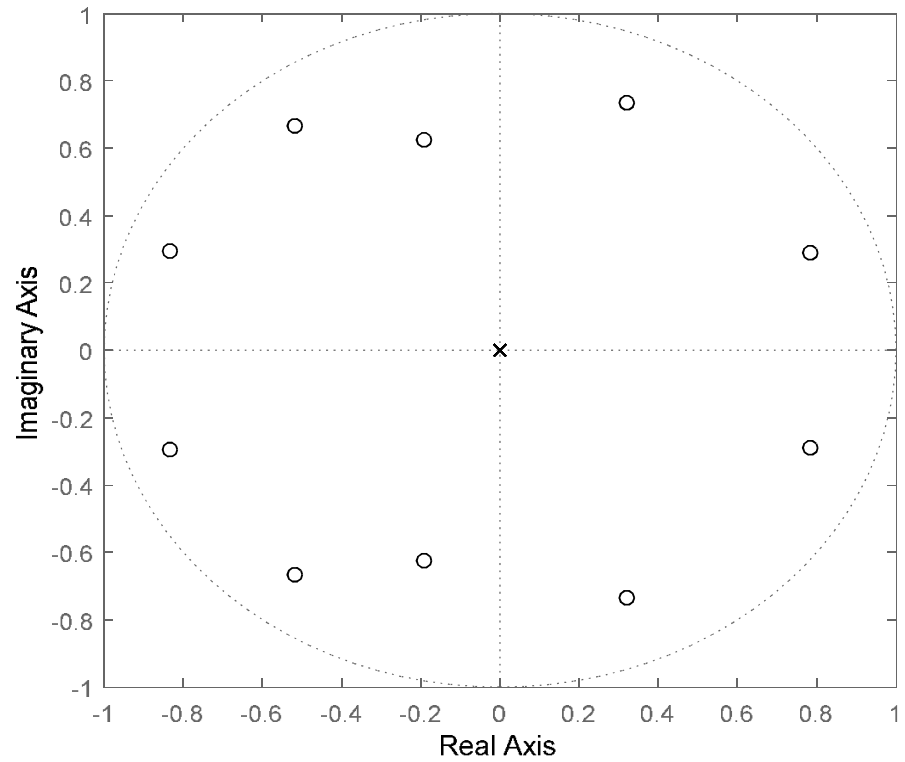

Figure 19. FIR-MRMN Filter Pole-Zero Plot for Signal Recovery from Impulsive Noise 


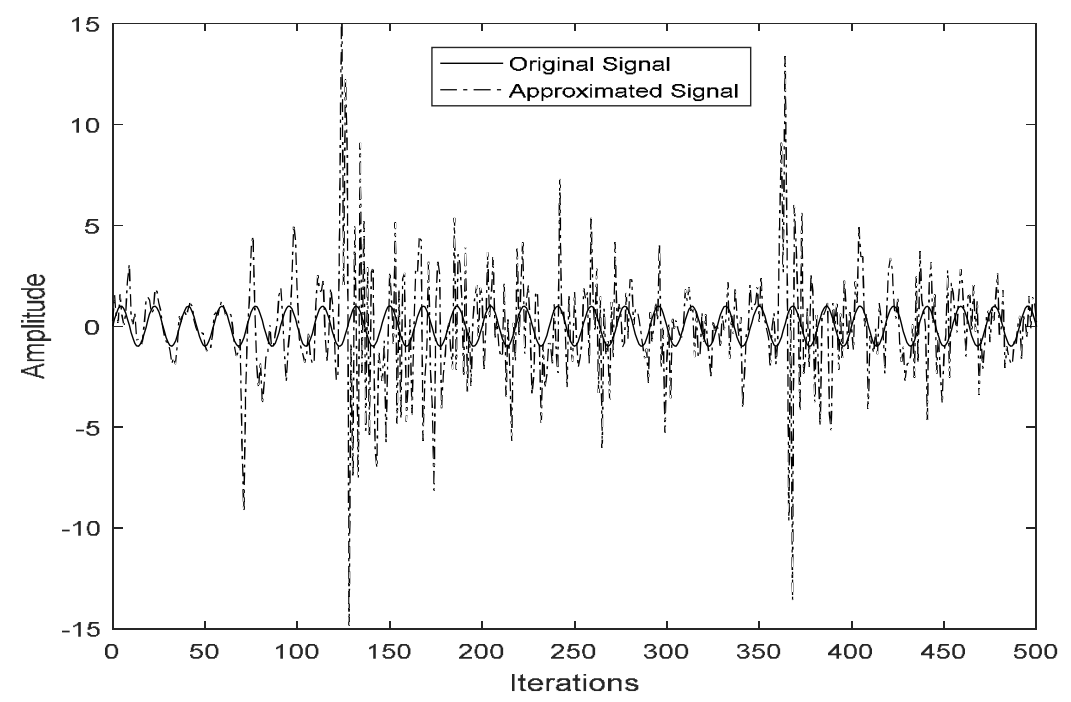

Figure 20. Signal Recovery from Impulsive Noise with IIR-LMS Filter

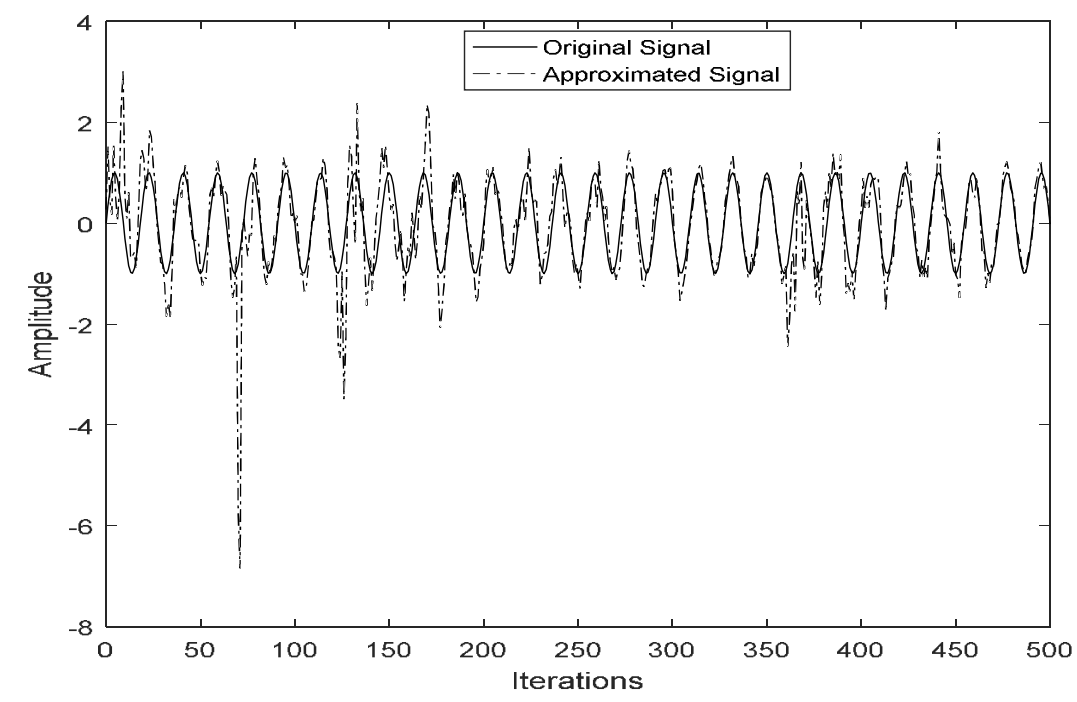

Figure 21. Signal Recovery from Impulsive Noise with IIR-MRMN Filter

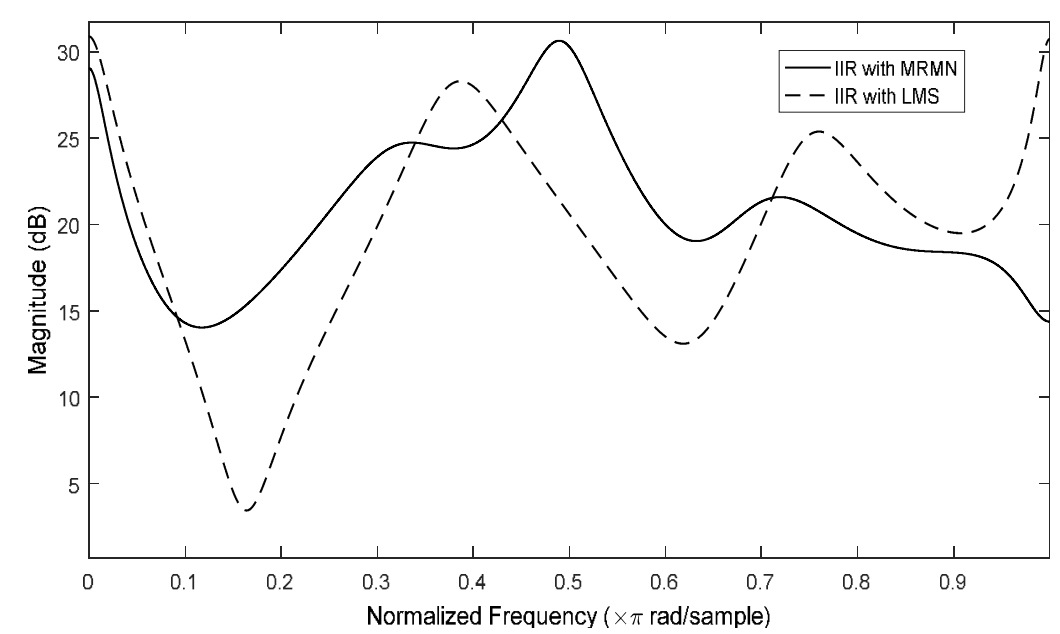

Figure 22. IIR Filter Response for Signal Recovery from Impulsive Noise 


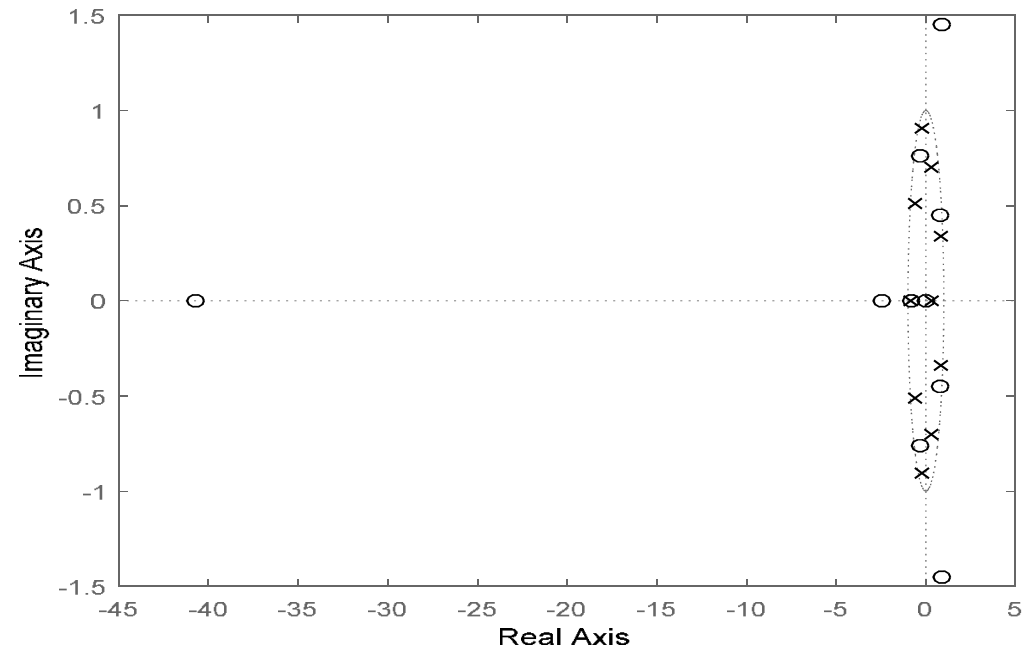

Figure 23. IIR-LMS Filter Pole-Zero Plot for Signal Recovery from Impulsive Noise

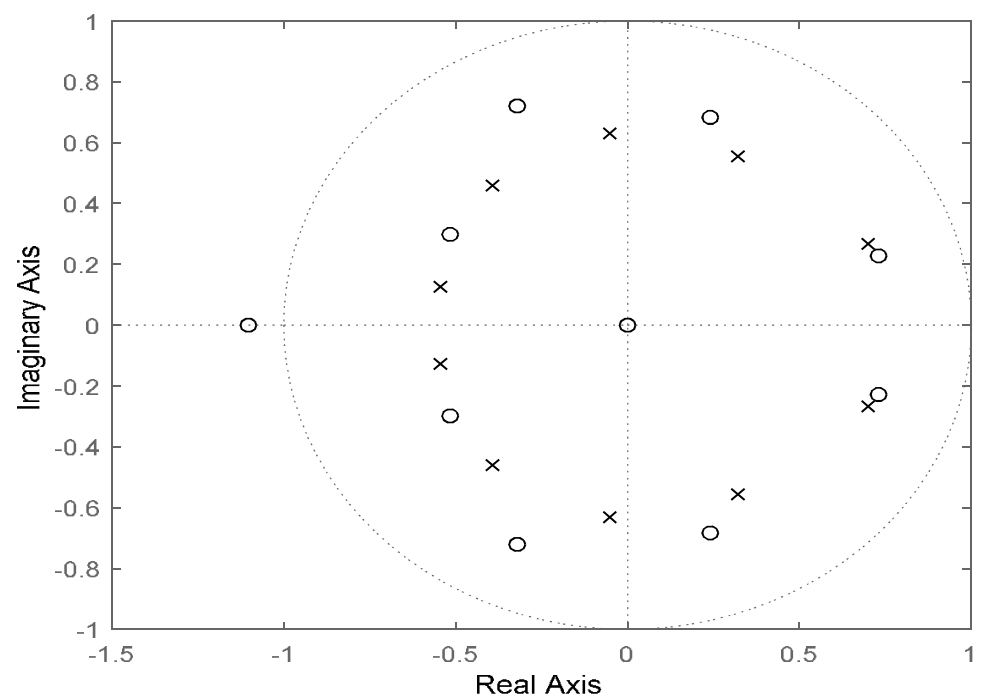

Figure 24. IIR-MRMN Filter Pole-Zero Plot for Signal Recovery from Impulsive Noise

Table 2: Percentage Signal Recovery and Recovery Speed from Impulsive Noise

$\begin{array}{ccc}\text { Filter Type } & \text { Percentage Signal Recovery } & \text { Speed of Recovery } \\ \text { FIR-LMS } & 25.7613 & 0.000511 \\ \text { FIR-MRMN } & 75.1684 & 0.005349 \\ \text { IIR-LMS } & 18.7239 & 0.001655 \\ \text { IIR-MRMN } & 75.76 & 0.006703\end{array}$

The results shown in Figures 14-24 and Table 2 gave an indication into the robustness of the MRMN algorithm in dealing with impulsive interference, as well as the relative advantage of the IIR configuration, most especially the proposed method, over other conventional approaches. The filters implementing the LMS algorithm could not compensate for the impulsive noise, and thus could not converge on the proper approximation of the original signal. The filters implementing MRMN algorithm however, were able to recover the original signal to a high level of confidence ( $75 \%$ ) and also compensate for the impulses, reducing some impulses, as seen around iterations $50-1500$, and then completely removing some, as seen around iterations $300-400$. It can also be seen again that the IIR filter implementing the MRMN algorithm had a better signal recovery than the FIR equivalent, due to its recursion, although the FIR filter was faster in execution.

The magnitude response graphs show the higher tendency for stability of the MRMN algorithm in the presence of impulsive interference, as the response of the MRMN algorithm filters tended to zero in a finite period, 
while the LMS filters were much higher and tended to infinity. This indicates that, the increment of impulses in the signal could completely diverge the LMS filters and their responses would go to infinity, hence causing instability, especially for the IIR LMS filter. The tendency for instability of the IIR filters can however be reduced or compensated for, by introducing more zeros into their transfer function, hence the feed forward cascade of the IIR filters are of the same order as the feedback filters, to provide adequate damping of all transients in response. Overall, the performance of all the filters with impulsive interference is described in terms of computational time, convergence, complexity and stability in Table 3.

Table 3: Performance Indicators for the All filters from Impulsive Noise

\begin{tabular}{|c|c|c|c|c|}
\hline Indicators & $\begin{array}{c}\text { IIR } \\
(\mathrm{MRMN})\end{array}$ & (LMS) & $\begin{array}{c}\text { FIR } \\
(\mathrm{MRMN})\end{array}$ & $\begin{array}{c}\text { FIR } \\
(\text { LMS) }\end{array}$ \\
\hline Computation time & Higher & Medium & High & Short \\
\hline Convergence & Better & Poorer & Good & Poor \\
\hline Stability & High & Low & Very High & High \\
\hline Complexity & Highest & Higher & High & Low \\
\hline
\end{tabular}

The computational complexity parameter is higher for the IIR filters, due to the need for recursive outputs in the computation of their current output values, since they require extra storage memory for keeping such delayed values of the output samples as opposed to the storage of only the input samples in the FIR implementation.

However, it should be noted that a higher order FIR filter implementation would be required to meet the convergence parameters, as well as signal approximation of the IIR filters implemented. The computational time parameter might however be negligible, due to the fast-computational speed and capacity of modern processors and computers in general, which has increased the processing ability of DSP chips and devices. The IIR filter with MRMN algorithm is recommended, based on the results presented and analysed in this report, for implementation in ANC applications that require more accuracy in signal recovery, especially with high level of impulsive interference.

\section{Conclusion}

A functional and stable IIR Adaptive filter for noise cancellation in signals, using a custom MRMN adaptive algorithm has been implemented through simulations. The developed system was compared with an equivalent IIR filter without the MRMN algorithm as well as FIR filters. It was discovered that the LMS algorithm diverged under impulsive noise conditions, while the MRMN algorithm could accommodate the impulsive interference, and thus the filters implementing the MRMN algorithm were better at signal recovery than the LMS filters. It was shown that the MRMN algorithm simply converges to the LMS algorithm under complete WGN interference, which indicates an accurate sensitivity of the algorithm to detection of impulses in the approximation process. The results give an indication of the possibility of stability, as well as a premise for practical realization/ adaptive IIR filtering, with the implementation of the MRMN algorithm to take care of sudden transients in the corrupted signals the filter might be required to approximate.

\section{References}

Apolinario Jr., J. A. \& Netto, S. L., 2009. Introduction to Adaptive Filters. s.1.:Springer.

Avalos, J. G., Sanchez, J. C. \& Velazq, J., 2011. Applications of Adaptive Filtering. In: Adaptive Filtering Applications, Dr Lino Garcia (Ed.). s.1.:InTech, pp. 1-20.

Chambers, J. A., Tanrikulu, O. \& Constantinides, A. G., 1994. Least Mean Mixed-Norm Adaptive Filtering. Electronics Letters, 30(19), pp. 1574-1575.

Chambers, J. \& Avlonitis, A., 1997. A Robust Mixed-Norm Adaptive Filter Algorithm. IEEE Signal Processing Letters, 4(2), pp. 46-48.

Chhikara, J. \& Singh, J., 2012. Noise Cancellation using Adaptive Algorithms. International Journal of Modern Engineering Research (IJMER), 3(2), pp. 792-795.

Dixit, S., 2017. LMS Adaptive Filters for Noise Cancellation: A Review. International Journal of Electrical and Computer Engineering (IJECE), 7(5), pp. 2520-2529.

Gupta, P. \& Beniwal, P., 2015. Adaptive Filters Algorithms: A Performance Comparison. International Journal of Engineering Research and General Science, 3(4), pp. 1-5.

Huang, Q., Chen, S., Huang, M. \& Guo, Z., 2017. Adaptive Active Noise Suppression Using Multiple Model Switching Strategy. Shock and Vibration, Volume 2017, pp. 1-6.

Lee, J.-H., Ooi, L.-E., Ko, Y.-H. \& Teoh, C.-Y., 2017. Simulation for noise cancellation using LMS adaptive filter. s.l., s.n.

Netto, S. L., Diniz, P. S. R. \& Agathoklis, P., 1995. Adaptive IIR Filtering Algorithms for System Identification: A General Framework. IEEE Transactions on Education, 38(1), pp. 54-66.

Parmar, C. A., Ramanadham, B. \& Darji, A. D., 2017. FPGA implementation of hardware efficient adaptive filter robust to impulsive noise. IET Computers \& Digital Techniques, 11(3), pp. 107-116. 
Papoulis, E. V. \& Stathaki, T., 2004. A Normalized Robust Mixed-Norm Adaptive Algorithm for System Identification. IEEE Signal Processing Letters, 11(1), pp. 56-59.

Qureshi, R., Uzair, M. \& Khurshid, K., 2017. Multistage Adaptive filter for ECG signal processing. Islamabad, Pakistan, s.n.

Rupp, M., 2015. Adaptive filters: stable but divergent. EURASIP Journal on Advances in Signal Processing, Volume (2015) 2015:104, pp. 1-15.

Stewart, B., 2006. Adaptive IIR Filtering. Birmingham, s.n.

Tanrikulu, O. \& Chambers, J. A., 1996. Convergence and Steady-State Properties of the Leasr-Mean Mixed Norm (LMMN) Adaptive Algorithm. IEE Proceeding - Vision Image and Signal Processing, 143(3), pp. 137-142.

Thakkar, V., 2017. Noise Cancellation using Least Mean Square Algorithm. International Journal of Engineering Research and Application, 7(10), pp. 46-57.

Upadhyay, P., Kar, R., Mandal, D. \& Ghoshal, S. P., 2016. A new design method based on firefly algorithm for IIR system identification problem. Journal of King Saud University - Engineering Sciences, Volume 28, pp. 174-198.

Vaseghi, S. V., 2000. Advanced Digital Signal Processing and Noise Reduction. s.1.:John Wiley \& Sons Ltd. 